\title{
Radio emission from polar caps in pulsars
}

\author{
Jan Kuijpers \\ Sterrekundig Instituut, P.O. Box 80.000, NL-3508 TA Utrecht, The \\ Netherlands \\ Martin Volwerk \\ Lunar and Planetary Laboratory, Univ. of Arizona, Tucson, AZ 85721, \\ $U S A$
}

\section{Physics of the proposed emission process}

Radiation from a charge accelerated along its path or Linear Acceleration Emission (LAE) involves a number of subtleties (Pauli 1921; Ginzburg 1970, 1989). Potential interest of the mechanism for astrophysics has been pointed out by Wagoner (1969). Melrose (1978) and Rowe (1995) have studied amplified LAE from time-varying electric fields for radio pulsars. In contrast with the latter work our calculations are for static electric field structures or double layers (DLs) as are thought to occur in magnetospheres of neutron stars. In ordinary stellar atmospheres a LAE maser can operate in non-relativistic DLs (Kuijpers 1990) at a frequency $\omega \approx k^{D L} v \approx 2 \pi / t_{t r}$, and a wave vector $\vec{k} \perp\left\{\vec{E}^{D L}, \vec{B}\right\}$ with $k^{D L}=2 \pi / L$ ( $L$ is the DL length, $v$ is the particle speed, and $t_{t r}$ is the transit time of the DL by the particle). The emission process can be considered as scattering of the electrostatic electric field on fast electrons into electromagnetic radiation satisfying the resonance condition: $\omega-\vec{k} \cdot \vec{v}=\omega^{D L}-\vec{k}^{D L} \cdot \vec{v} \approx-\vec{k}^{D L} \cdot \vec{v}$, when the frequency of the radiated mode in the frame of the emitting electron equals the Doppler shifted frequency of the electric field of the DL (DL wave frequency $\omega^{D L} \approx 0$ ). For relativistic DLs, as are applicable to pulsar magnetospheres, the emission is expected to be beamed under an angle $\theta \approx \gamma^{-1}$ and the frequency of emission boosted $\left(\omega \approx k^{D L} v(1-v \cos \theta / c)^{-1} \approx \gamma^{2} k^{D L} v\right)$.

\section{Approximations}

We model the DL by a static, localized, uniform electric field $\vec{E}_{0}$ antiparallel to the magnetic field $\vec{B}$. We neglect pair creation and choose an initial onedimensional electron distribution with positive slope $p$ entering the DL: $f(\gamma)=$ $N \gamma^{p}(p+1) /\left(\gamma_{1}^{p+1}-\gamma_{0}^{p+1}\right), \quad \gamma_{0} \leq \gamma \leq \gamma_{1}$. We treat the electromagnetic wave as a pure vacuum wave, and perturb around the unperturbed particle trajectory $(v \approx c)$. We assume that $\left|\vec{k} \cdot \vec{r}^{(1)}(t)\right|<\pi / 2$ and $|\vec{k} \cdot \ddot{\vec{r}}(t)| \gg\left|\vec{k} \cdot \vec{v}^{(1)}\right|^{2}$ over the integration interval, which, in terms of the initial and, respectively, final Lorentz factors $\gamma_{\mathrm{i}}, \gamma_{\mathrm{f}}$, implies $k \lesssim \pi \gamma_{\mathrm{i}}^{2} /(L \cos \theta), k<4 \gamma_{\mathrm{i}}^{4} \gamma_{\mathrm{f}} /\left[\gamma^{3}(t) L \cos \theta\right]$, where $\theta$ is the angle between $\vec{k}$ and $\vec{v}$. Since the acceleration between $t=0$ and $t=t_{\mathrm{tr}}$ $\left(t_{\mathrm{tr}} \simeq L / c\right.$ is the transit time for an electron) strongly decreases with the Lorentz 
factor $\left(\propto \gamma^{-3}\right)$, while the Lorentz factor in the relativistic regime changes as $\gamma(t)=\gamma_{i}\left(1+\frac{e E_{0}}{\gamma_{i} m_{e} c} t\right)=\gamma_{i}\left(1+t / \tau_{0}\right)$, we limit the integration in the first-order current density to the interval $t=0$ to $t=\tau_{0} \equiv \gamma_{\mathrm{i}} m_{\mathrm{e}} c / e E_{0}$. We also take the limit of small angles with respect to the electric field: $\theta \simeq \gamma_{\mathrm{i}}^{-1}\left(c / v^{(0)}\right)^{1 / 2}$ where the incoherent emission is boosted with a factor $\gamma_{i}^{10} c / v^{(0)}\left(\gamma_{i}\right.$ and $v^{(0)}$ are related by $\left.v^{(0)}=c\left(1-\gamma_{\mathrm{i}}^{-2}\right)^{1 / 2}\right)$. Finally we assume that amplification conditions remain constant along a ray path inside the DL.

\section{Results}

We find that coherent radio emission can be produced at frequencies $\omega / 2 \pi \lessgtr$ $0.5 \gamma_{i}^{2} / \tau_{0}$, where $\gamma_{i}$ is the Lorentz factor of the particles entering the DL and $\tau_{0}=\gamma_{i} m_{e} c / e E_{0}$ is their characteristic acceleration time. The radiation occurs at an angle $\gamma^{-1}$ with respect to the particle orbit (the magnetic field). As the electric field in the DL is maintained by the rotation of the magnetized neutron star, it remains constant on the time scale of the emission process and the mechanism can be considered a true maser. With the usual Rayleigh-Jeans definition of the brightness temperature we finally arrive at

$$
T_{\mathrm{b}}(\omega)=-\frac{m_{\mathrm{e}} c^{2}}{k_{\mathrm{B}} p} \frac{\Gamma_{6}}{\Gamma_{5}}\left(1-\exp \left\{\frac{4 \pi e^{4} E_{0}^{2} n_{\gamma} p}{m_{\mathrm{e}}^{3} c^{3} \omega^{4}}\left(\frac{\gamma}{\tau_{0}}\right) \Gamma_{5} L\right\}\right) \mathrm{K},
$$

where we have used an amplification length $s=L / \cos (1 / \gamma) \approx L, n_{\gamma}=N(p+$ 1) $/\left(\gamma_{1}^{p+1}-\gamma_{0}^{p+1}\right), \tau_{0} / \gamma=m_{e} c / e E_{0}, \Gamma_{5}=\left.\bar{\gamma}^{-2}(p+5)^{-1} \gamma^{p+5}\right|_{\gamma_{0}} ^{\gamma_{1}}$ and $\Gamma_{6}=\bar{\gamma}^{-2}(p+$ $6)\left.^{-1} \gamma^{p+6}\right|_{\gamma_{0}} ^{\gamma_{1}}$. We have studied the dependence of the brightness temperature of LAE on various parameters (Volwerk and Kuijpers 1996). We find that brightness temperatures of $10^{31} \mathrm{~K}$ at radio frequencies can be obtained from electron beams with small Lorentz factors ( $\gamma$ a few).

\section{References}

Ginzburg, V.L. 1970, Sov. Phys. Usp. 12, 565

Ginzburg, V.L. 1989, Applications of Electrodynamics in Theoretical Physics and Astrophysics, Gordon \& Breach Science Publishers, New York

Kuijpers, J. 1990, in: Plasma Phenomena in the Solar Atmosphere, 1989 Cargèseworkshop, eds. M.A. Dubois, F. Bély-Dubau and D. Gresillon, Les Éditions de Physique, Les Ulis, France, p. 1

Melrose, D.B. 1978, ApJ225, 557

Pauli, W. 1921: see Pauli, W. 1981, Theory of Relativity, Dover Public., New York, $\S 32(\gamma)$

Rowe, E.T., 1995, A\&A296, 275

Volwerk, M. and Kuijpers, J. 1996, in preparation for A\&A

Wagoner, R.V. 1969, ApJ158, 739 